\title{
TeV $\gamma$-radiation from Dark Matter annihilation in the Galactic center
}

\author{
D. Horns \\ Max-Planck-Institut für Kernphysik, Postfach 1039 80, D-69029 Heidelberg, \\ Germany
}

\begin{abstract}
Recent observations of the Galactic center with ground based gamma-ray instruments have revealed a $\mathrm{TeV}\left(10^{12} \mathrm{eV}\right)$ gamma-ray signal consistent with the position of Sgr A*. The origin of the recently established $\mathrm{TeV}$ gamma-ray signal is so far unclear. The derived luminosity of the signal above $1 \mathrm{TeV}$ is a few $10^{34} \mathrm{erg} \mathrm{s}^{-1}$ which is slightly more than e.g. the gamma-ray luminosity of the Crab nebula and a plausible identification could be a conventional albeit strong gamma-ray source. The Galactic center region covered by the $\mathrm{TeV}$ error box hosts a large number of known sources of non-thermal radiation including Sgr A East and Sgr A* which could produce collectively or in outbursts sufficient luminosity to explain the observed flux. The observations with the H.E.S.S. system of Cherenkov telescopes have decreased the solid angle subtended by the error box by more than a factor of hundred with respect to the previous observations of the VERITAS and CANGAROO groups. Even though no clear evidence like a line feature in the spectrum is observed, at this point there is also no clear evidence (like variability) which would rule out a Dark Matter interpretation. After studying the observed energy spectrum and angular distribution of the excess as seen by the H.E.S.S. experiment, a massive Dark Matter candidate with a minimum mass of $12 \mathrm{TeV}(90 \%$ c.l. $)$ and an upper limit on the WIMP density for $r<10 \mathrm{pc}$ of $1261 M_{\odot} \mathrm{pc}^{-3} \times\left(\langle\sigma v\rangle / 3 \cdot 10^{-26} \mathrm{~cm}^{3} \mathrm{~s}^{-1}\right)^{-1 / 2}$ is required to explain the observed flux by an annihilation signal. The angular distribution of the excess events is consistent with a cuspy profile with $\rho(r) \propto r^{-\alpha}$ with $\alpha>1.0$ at a confidence level of $90 \%$. Even though the mass and the cross section of the Dark Matter constituents are unexpectedly high in the framework of most models of nonbaryonic Dark Matter, it can not be ruled out.
\end{abstract}

Key words:

PACS: 95.35.+d, 98.35.Jk, 98.35.Gi, 95.85.Pw, 96.40.Pq 


\section{Introduction}

The presence of a Dark Matter halo of the Galaxy is observationally established from stellar dynamics [1]. At present, the nature of Dark Matter is unfortunately unknown but a number of viable candidates have been advocated within different theoretical frameworks mainly motivated by particle physics (for recent reviews see e.g. [2,3]) including the widely studied models of Supersymmetric (SuSy) Dark Matter [4]. On the cosmological scale, the presence of non-baryonic and predominantly cold Dark Matter as it had been suggested in [5] is well established by many observations most notably of the WMAP experiment [6].

The implications of the possible presence of SuSy Dark Matter in the Galactic center has been studied in numerous publications including predictions for the $\gamma$-ray flux expected from annihilation of weakly interacting massive particles - WIMPs (see e.g. $[7,8,9,10,11]$ ). With the advent of the next generation of Cherenkov telescopes a gamma-ray signal from the direction of the Galactic center has been established: The recent claims for gamma-rays from the Galactic center by the CANGAROO collaboration with a signal $S=9.8 \sigma$ [12] and a possible excess claimed by the VERITAS collaboration on the level of $3.7 \sigma$ [13] have already stimulated first speculations on the interpretation of the signal as annihilation radiation from Dark Matter [14]. Finally, the recently commissioned H.E.S.S. system of Cherenkov telescopes has detected a signal from the vicinity of $\operatorname{Sgr} A^{*}[15]$. The different confidence regions of the detections by the different instruments in comparison with a $21 \mathrm{~cm}$ radio map [16] are indicated in Fig. 1 and the flux measurements are given in Fig. 2. Note, the EGRET measurements taken from $[17,18]$ give the range of possible flux values for non-resolved objects in the gamma-ray background dominated region and should be interpreted as upper limits for a contribution of Sgr A*. The upper limit indicated in Fig. 2 at $1 \mathrm{GeV}$ is from a dedicated analysis of the high energy EGRET data [19] assuming a photon index of 1.5.

At first glance, the differences in the reported $\mathrm{TeV}$ flux by the CANGAROO and H.E.S.S. groups might suggest variability at the lower end of the energy spectrum thus ruling out an exclusive annihilation origin and hinting at a more conventional compact gamma-ray source related to Sgr A*. However, given the positional uncertainty of the CANGAROO II and VERITAS results evident in Fig. 1, it remains possible that in fact more than one (possibly even variable) source may exist in the direction of the Galactic center. Interestingly, the observations of H.E.S.S. indicate the presence of even further sources in the field of view (see Fig. 1 of [15]).

Further observations with H.E.S.S. and CANGAROO III in the coming years will help to clarify the situation which might be consistent with multiple 
sources which are not resolvable by single telescope observations. Instrumentally, the CANGAROO and H.E.S.S. observations have shown discrepancies in the flux values for other steady sources: SN1006, PSR B1706-44, Vela, and RXJ1713-304 [20,21,22] - a similar situation might be present in this case.

The VERITAS energy flux given in Fig. 2 is converted from the $40 \%$ Crab flux as measured with the Whipple telescope, assuming a photon index of 2.2 , and using the Crab flux as determined by H.E.S.S. to compare the two instruments' observations. The error bars are given as the sum of statistical and systematic uncertainties. The two measurements are in reasonable agreement and given the possible positive bias in the flux estimate for a source detected at the level of less than 4 standard deviations should be considered to be consistent within the uncertainties.

The discussion here is focussing on the results obtained with the H.E.S.S. system of telescopes which achieves a superior angular resolution in comparison to the previously published results as is evident from Fig. $1^{1}$. The reconstructed source location is, within the errors, consistent with the position of Sgr A*(space angle $\Delta \theta=(18 \pm 42) ")$ and appears to be consistent with a pointsource for the given angular resolution of 5.8' (50\% containment radius $[15])$.

The H.E.S.S. observations (spectrum and angular distribution) are interpreted in the framework of a Dark Matter annihilation scenario to investigate the consequences for the WIMP mass, density, and spatial structure and to understand whether such an interpretation is feasible.

\section{WIMP mass}

In order to quantify the minimum mass required to explain the observed energy spectrum in terms of an annihilation signal, an assumption on the shape of the annihilation continuum spectrum is required. In the very generic case of a self-annihilating massive WIMP, it is assumed that the branching ratio for $q \bar{q}, W^{+} W^{-}$, and $Z^{0} Z^{0}$ is quite large.

Subsequent hadronization leads to the production of charged and neutral mesons which decay finally into electrons, neutrinos, and gammas. The gamma signal is described by a fairly hard differential energy spectrum with an exponential cut-off $d \Phi / d E \propto E^{-1.5} \exp \left(-E / E_{c}\right)$ with $E_{c} \approx 0.1 m_{\chi}$ [7]. However, the exact shape of the spectrum depends on details of the branching ratio of

$\overline{1}$ These data were taken during the build-up phase and will eventually be superseded by the performance of the full telescope array 


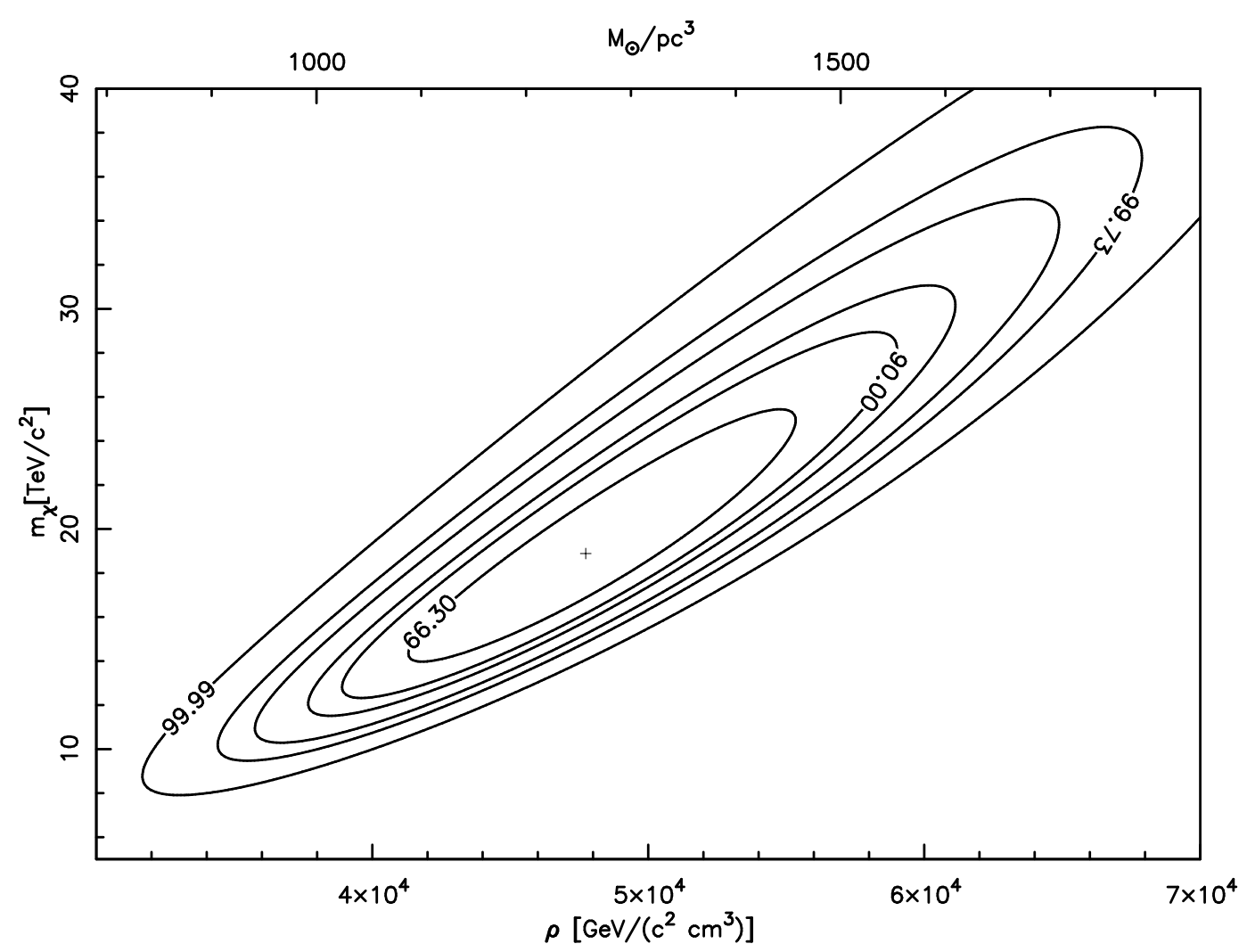

Fig. 1. Position of the TeV emitting region overlaid on a $21 \mathrm{~cm}$ radio map [16] tracing mostly non-thermal (synchrotron) emission features. The bright spot inside the H.E.S.S. confidence region (marked as a white circle) is $\mathrm{Sgr} \mathrm{A}^{*}$ whereas the extended ring like feature to the east of it is Sgr A*East. Note the difference in the accuracy of the different instruments.

other processes. Namely, for $\chi \chi \rightarrow \tau \bar{\tau}$, direct radiative losses produce a second hard component.

For the purpose of calculating a lower limit on $m_{\chi}$ it is assumed that the branching ratio for $\chi \chi \rightarrow q \bar{q}$ or $W^{+} W^{-}$is close to one. The minimum mass requirement is somewhat relaxed if $\chi \chi \rightarrow \tau \bar{\tau}$ is of importance.

Fitting a generic $\pi^{0}$ decay spectrum to the data results in a $90 \%$ c.l. lower limit on the mass $m_{\chi}$ to be larger than $12 \mathrm{TeV}$.

In most models that attempt to explain non-baryonic Dark Matter in the Universe, the relic particles are WIMPs produced thermally during the early phase of cosmological expansion. A very reasonable candidate for this particle is provided by SuSy models which naturally predict the existence of a stable (if R-parity is conserved) Majorana particle that can self-annihilate. Generically, this SuSy particle is referred to as the lightest stable particle (LSP).

The detection of gamma-rays with energies up to $10 \mathrm{TeV}$ as seen with the H.E.S.S. telescopes implies that the WIMP mass has to be sufficienly large 
to explain the observed spectrum. The surprisingly large mass inferred from the observations appears at first glance to be inconsistent with cosmology and the general conception that the mass of LSP in SuSy models should be close to the electro-weak unification scale [23]. However, it has been argued that a multi-TeV WIMP is in fact natural in Minimal Supergravity models [24]. The upper bound on the possible mass of a WIMP that decoupled thermally during the early phase of expansion is around $40 \mathrm{TeV}$ [25] for a cosmological non-baryonic Dark Matter density of $\Omega_{d m} h^{2}=0.1$.

The average annihilation cross section $\langle\sigma v\rangle$ for SuSy WIMPs is in the right range to explain today's Dark Matter density as determined by the WMAP mission: $\Omega_{d m} h^{2}=0.116 \pm 0.020$ [6]. However, given that for the thermal decoupling $\Omega_{d m} h^{2} \propto\langle\sigma v\rangle^{-1}$ and that $\langle\sigma v\rangle \propto m_{\chi}^{-1}$, rather tight constraints on the mass of the WIMP $m_{\chi}<600 \mathrm{GeV}$ can be derived [26]. However, as already pointed out in [26] there are specific manifestations of SuSy models (even in the constrained, universal models) that allow for larger WIMP masses which still produce the right relic density:

(1) For specific SuSy models in which the LSP is almost degenerate in mass with other SuSy particle (e.g. $\tilde{\tau}$ ) coannihilation depletes the relic density even for a small annihilation cross section.

(2) For a non-thermal decoupling process, the relic density as it is today is not depending on the cross section.

(3) Relaxing the universality of the Higgs multiplett masses at the GUT scale: In this (and in the general SuSy) case, it is conceivable that a massive (multi-TeV) WIMP with the right relic density may exist. Thess WIMPs are generally almost pure Higgsino type Neutralinos with a large cross section.

In the first case, the cross section for annihilation remains small and therefore requires high relic density values in the Galactic center to explain the observed $\gamma$-ray flux. In the special case of a degeneracy between the LSP and other SuSy particles, careful tuning is required to achieve the right relic density. For a massive LSP beyond a few TeV, even co-annihilation does not suffice to reduce the relic density. A non-thermal scenario invoking Q-balls [27] which replenish the Dark Matter content via evaporation at some time after the decoupling has been studied in the literature [28,29]. In this and other nonthermal scenarios the cross section for WIMPs could be rather high.

The last case is more attractive but again requires tuning of the Gaugino masses at the unification scale which might appear less attractive from the point of view of a natural theory.

Generally, a massive WIMP as suggested by the observations with a sufficiently high cross section is not the obvious candidate in the framework of 
MSUGRA but could be the LSP in slightly less general theories. In this way, thermal decoupling would produce the right relic density and the observed flux could be consistent with a Dark Matter density which is not violating other astrophysical bounds.

Ultimately, a neutralino mass exceeding $40 \mathrm{TeV}$ is excluded by the unitarity bound in the framework of thermal decoupling [25].

\section{WIMP density}

Given the measured flux, it is possible (without assuming a specific halo shape) to derive an upper limit on the product $\left\langle\rho_{d m}\right\rangle_{r<R_{0}} \sqrt{\langle\sigma v\rangle}$ This is truly an upper limit, as can be seen from the following argument:

Assuming that the true density distribution is spherically symmetric and follows a general form [7]

$$
\rho(r)=\frac{1}{(r / a)^{\gamma}\left[1+(r / a)^{\gamma}\right]^{(\beta-\gamma) / \alpha}}
$$

The halo density profiles predicted from N-body simulations of hierarchical structure formation follow a power law roughly between $\gamma=1$ [30] and $\gamma=1.5$ [31]. The annihilation flux observed from within the Galaxy is proportional to the line of sight integral

$$
J(\psi)=J_{0} \times \int_{\text {l.o.s. }} d l \rho^{2}\left(\sqrt{R^{2}-2 l R \cos \psi+l^{2}}\right)
$$

averaged over the solid angle observed:

$$
\langle J(0)\rangle_{\Delta \Omega} \Delta \Omega=J_{0} \times \int d \Omega \int d l \rho^{2}
$$

with the commonly used normalization for the Galactic Center:

$$
J_{0}=\frac{1}{8.5 \mathrm{kpc}} \frac{1}{\left(0.3 \mathrm{GeV} \mathrm{cm}^{-3}\right)^{2}}=4.2 \times 10^{-22} \mathrm{GeV}^{-2} \mathrm{~cm}^{5}
$$

For reasons of simplification and in order to calculate an upper limit, it is convenient to use a truncated version of Eq. 1 which drops to zero outside a spherical volume with radius $R_{0}$. Obviously, the derived density to match an observed flux would be higher than in the case of a continuous halo distribution. For a truncated density function with $R_{0}$ much smaller than the distance 
of the observer to the sun, the line of sight integral can be safely replaced by a volume integral and the observed differential gamma-ray flux is given by:

$$
\begin{aligned}
\frac{d \Phi}{d E} & =\frac{1}{2} \frac{1}{4 \pi d^{2}} \frac{d N}{d E} \frac{\langle\sigma v\rangle}{m_{\chi}^{2}} \times \int d V \rho^{2} \\
& \propto\left\langle\rho^{2}\right\rangle V
\end{aligned}
$$

Considering a truncated density function which follows a single power law up to a radius $R_{0}$ dropping to zero for larger radii: $\rho(r)=\rho_{0} \times\left(R_{0} / r\right)^{\alpha}$ with $\alpha=0 \ldots 1.5$.

$$
\begin{aligned}
\langle\rho\rangle & =\frac{\int d V \rho}{\int d V} \\
& =\rho_{0} \times \frac{3}{3-\alpha}
\end{aligned}
$$

and for $\left\langle\rho^{2}\right\rangle$ :

$$
\begin{aligned}
\left\langle\rho^{2}\right\rangle & =\frac{\int d V \rho^{2}}{\int d V} \\
& =\rho_{0}^{2} \times \frac{3}{3-2 \alpha}
\end{aligned}
$$

which gives for the ratio $\left\langle\rho^{2}\right\rangle /\langle\rho\rangle^{2}$ :

$$
\frac{\left\langle\rho^{2}\right\rangle}{\langle\rho\rangle^{2}}=\frac{(3-\alpha)^{2}}{3(3-2 \alpha)}
$$

This ratio is $>1$ for $\alpha>0$. For $\alpha=0$ the trivial case of a constant density and $\left\langle\rho^{2}\right\rangle=\langle\rho\rangle^{2}$ is recovered. For $\alpha>0\left\langle\rho^{2}\right\rangle>\langle\rho\rangle^{2}$ which implies for a given flux $\left(\propto\left\langle\rho^{2}\right\rangle\right.$, see Eq. 5) a lower average density than in the case of a constant WIMP density ${ }^{2}$. Therefore, calculating an average density with $\alpha=0$ from the observed flux sets an upper limit on the actual average density for a realistic radial density distribution.

For convenience, Eq. 5 can be rewritten with appropriate units:

$$
\frac{d \Phi}{d E}=0.54 \times 10^{-12}\left(\mathrm{~cm}^{2} \mathrm{~s}\right)^{-1} \frac{\langle\rho\rangle^{2}}{\left(5000 \mathrm{GeV} \mathrm{c} c^{-2} \mathrm{~cm}^{-3}\right)^{2}} \cdot \frac{\langle\sigma v\rangle}{3 \times 10^{-26} \mathrm{~cm}^{3} \mathrm{~s}^{-1}}
$$

2 For the sake of brevity, this is only shown for $0<\alpha<1.5$, but it holds true for any $\alpha>0$. 


$$
\left(\frac{m_{\chi}}{\mathrm{TeV}}\right)^{-2} \times \frac{(3-\alpha)^{2}}{3(3-2 \alpha)} \times\left(\frac{d}{8.5 \mathrm{kpc}}\right)^{2} \times\left(\frac{R_{0}}{10 \mathrm{pc}}\right)^{3} \frac{d N}{d E}
$$

In the more general case of a radial density profile extending beyond $R_{0}$, the line-of-sight integral of Eq. 3 will naturally give larger observed fluxes $(\propto$ $\langle J(0)\rangle_{\Delta \Omega} \Delta \Omega$ ) than for a truncated density therefore relaxing the requirement of the average central density $\langle\rho\rangle$ even more. In this sense, the approach of calculating an average, truncated density is truly an upper limit on the average density as expected for a realistic, smooth Dark Matter halo distribution.

The parameterization for the decay spectrum is of course depending on the nature of the WIMP and cannot be given in general. For the case of a very massive WIMP considered here, the dominant annihilation channel is into a $W^{+} W^{-}, Z^{0} Z^{0}$, and $q \bar{q}$ pairs. A parameterization of the form

$$
\frac{d N}{d E}=\frac{0.73}{m_{\chi}} \times \frac{e^{-7.8 E / m_{\chi}}}{\left(E / m_{\chi}\right)^{1.5}+E_{0}}
$$

is a reasonable description of the decay spectrum (with $E$ and $m_{\chi}$ in $\mathrm{TeV}$, $\left.E_{0}=2 \cdot 10^{-4} \mathrm{TeV}\right)$.

In order to calculate the upper limit on $\langle\rho\rangle$ it is assumed that the entire observed flux is produced by annihilation of WIMPs. The H.E.S.S. data-points are taken from [15] with the assumed emission region being covered by the point-spread function of $\approx 5.8^{\prime}$. The actual limit on the source size is only 3'. However, this assumes a Gaussian surface emission profile, whereas a volume source with constant emissivity is expected. The upper limit on such an emission profile will be somewhat bigger than 3' but smaller than the point spread function. The upper limit depends on the assumed emission region as $\langle\rho\rangle \propto R_{0}^{-3 / 2}$.

The H.E.S.S. data points (open triangles) are indicated in Fig. 2 together with the CANGAROO (open boxes), VERITAS (open diamond), and different EGRET flux values (open symbols from [17], filled symbols from [18], and upper limit from [19]). A best fit model of the H.E.S.S. data requires

$$
\begin{aligned}
m_{\chi} & =(18.9 \pm 3.7) \mathrm{TeV} \\
\langle\rho\rangle_{r<10 \mathrm{pc}} & =(47900 \pm 4700) \frac{\mathrm{GeV}}{\mathrm{c}^{2} \mathrm{~cm}^{3}} \times\left(\frac{\langle\sigma v\rangle}{3 \times 10^{-26} \mathrm{~cm}^{3} \mathrm{~s}^{-1}}\right)^{-1 / 2} \frac{\sqrt{3(3-2 \alpha)}}{3-\alpha} \\
& =(1261 \pm 124) \frac{M_{\odot}}{\mathrm{pc}^{3}} \times\left(\frac{\langle\sigma v\rangle}{3 \times 10^{-26} \mathrm{~cm}^{3} \mathrm{~s}^{-1}}\right)^{-1 / 2} \frac{\sqrt{3(3-2 \alpha)}}{3-\alpha}
\end{aligned}
$$

with $\chi_{\text {red }}^{2}($ d.o.f. $)=1.43(13)$. 
The confidence regions given by the $\chi^{2}$ minimization for the two parameters $\langle\rho\rangle$ and $m_{\chi}$ is given in Fig. 3. The best fit position is indicated by a small cross.

For the sake of completeness, a similar procedure can be applied to the CANGAROO data giving the following values:

$$
\begin{aligned}
m_{\chi} & =(1.1 \pm 0.3) \mathrm{TeV} \\
\langle\rho\rangle_{r<47 \mathrm{pc}} & =(7840 \pm 1170) \frac{\mathrm{GeV}}{c^{2} \mathrm{~cm}^{3}} \times\left(\frac{\langle\sigma v\rangle}{3 \times 10^{-26} \mathrm{~cm}^{3} \mathrm{~s}^{-1}}\right)^{-1 / 2} \frac{\sqrt{3(3-2 \alpha)}}{3-\alpha} \\
& =(206 \pm 31) \frac{M_{\odot}}{\mathrm{pc}^{3}} \times\left(\frac{\langle\sigma v\rangle}{3 \times 10^{-26} \mathrm{~cm}^{3} \mathrm{~s}^{-1}}\right)^{-1 / 2} \frac{\sqrt{3(3-2 \alpha)}}{3-\alpha}
\end{aligned}
$$

with $\chi_{\text {red }}^{2}$ (d.o.f. $)=0.81(4)$. The value given for the density is higher than calculated in [12] because of the factor of 1/2 in Eqn. 5 that has been neglected in the past [32]. As pointed out in the Introduction, the CANGAROO-II result is not consistent with the H.E.S.S. observations hinting at either a mismatch in the cross-calibration of the two instruments or a variable object which might coincide with one of the more conventional gamma-ray sources close to the Galactic center.

The required average density depends on the cuspiness of the halo profile and scales according to Eqn. 9 with $\sqrt{3(3-2 \alpha} /(3-\alpha)$ (for $\alpha<1.5)$ and is reduced by a factor of 2 with respect to a constant density halo distribution when $\alpha \approx 1.4$.

\section{Spatial WIMP distribution}

Given the excellent angular resolution of the H.E.S.S. telescope arrays of 5.8' for individual events [15], spatial extension of the source down to this level can be probed. The signal appears to be point-like (i.e. of spatial extension smaller than the point spread function) with an upper limit of 3' for a source with a Gaussian surface brightness profile.

The expected source extension from Dark Matter annihilation depends crucially on the shape of the radial density profile. For a quantitative study, a simplification of the radial density profile given in Eqn. 1 is applied:

$$
\begin{aligned}
\rho(r) & =\rho_{0} \times\left(\frac{a}{r}\right)^{\alpha}(r<a=8.5 \mathrm{kpc}) \\
& =0(r>a)
\end{aligned}
$$




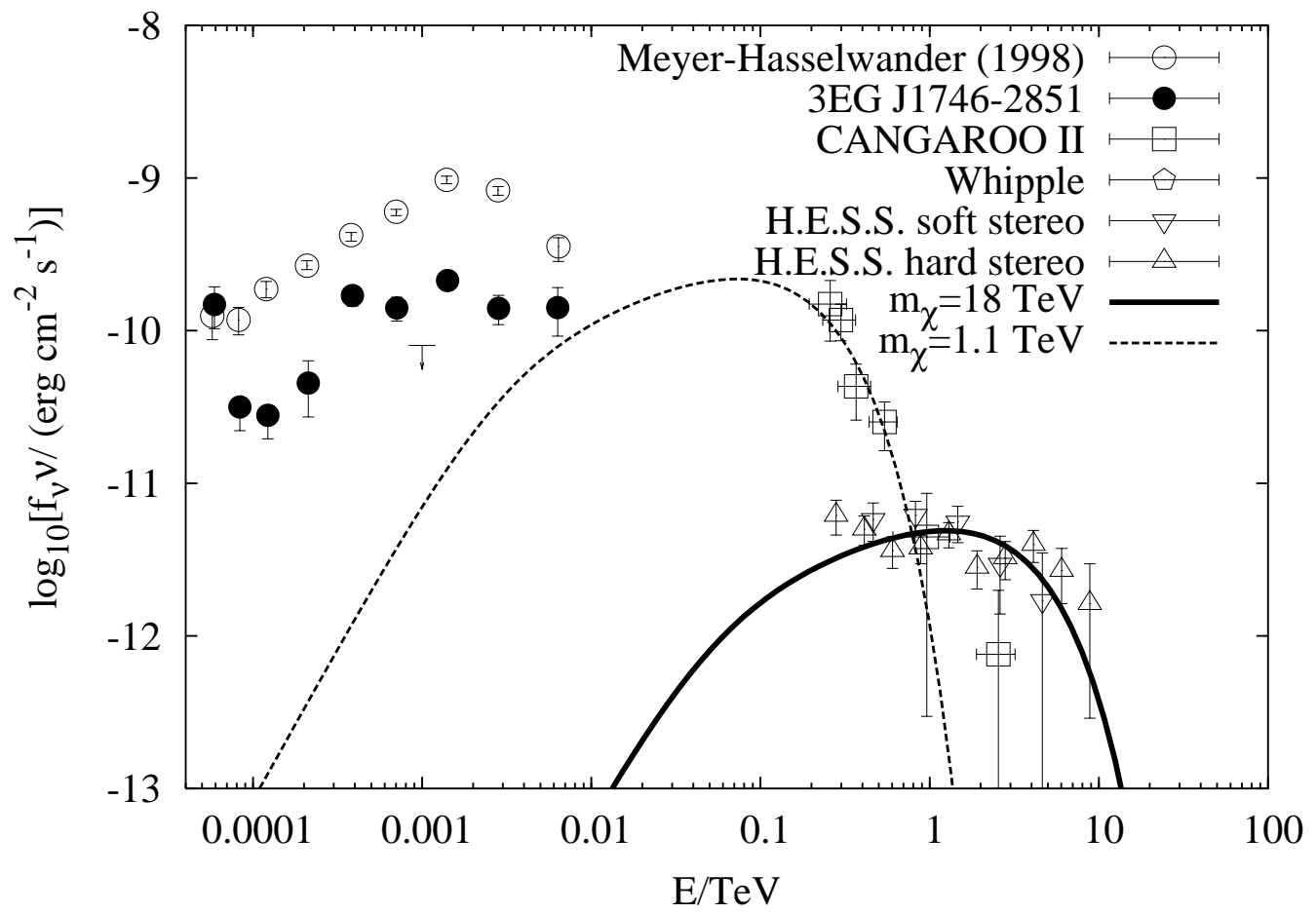

Fig. 2. A summary of data and best-fit models for WIMP annihilation from the Galactic center: H.E.S.S. (open triangles), CANGAROO (open boxes), EGRET (solid and open circles), 10m Whipple telescope of the VERITAS collaboration (solid diamond).

Since the observations cover only the inner $0.5^{\circ}$ of the Galactic center (equivalent to $75 \mathrm{pc}$ ), the actual value of $a$ is not crucial.

The observed surface brightness is calculated by folding $J(\psi)$ (Eqn. 2) with the average point spread function of H.E.S.S. for the observations approximated by a double Gaussian

$$
f_{p s f}(\psi)=f_{0} \cdot\left(\exp \left(-0.5 \psi^{2} / \sigma_{1}^{2}\right)+1 / 8.7 \cdot \exp \left(-0.5 \psi^{2} / \sigma_{2}^{2}\right)\right)
$$

with $\sigma_{1}=0.052^{\circ}, \sigma_{2}=0.136^{\circ}$ (J. Hinton, priv. communication), and $f_{0}$ as an arbitrary normalization:

$$
p(\psi)=p_{0} \times \int_{-\infty}^{\infty} d \psi^{\prime} \cdot f_{p s f}\left(\psi-\psi^{\prime}\right) J\left(\psi^{\prime}\right)
$$

with $p_{0}$ chosen such that $\int d \psi p(\psi)=1$. The resulting angular distribution is shown in Fig. 4 for different values of $\alpha$. Using the $\chi^{2}$ values for various $\alpha$, a $90 \%$ c.l. (one sided) for $\alpha>1$ is calculated which is suggestive of a halo with a halo steeper than predicted by Navarro, Frenk, and White [30]. 


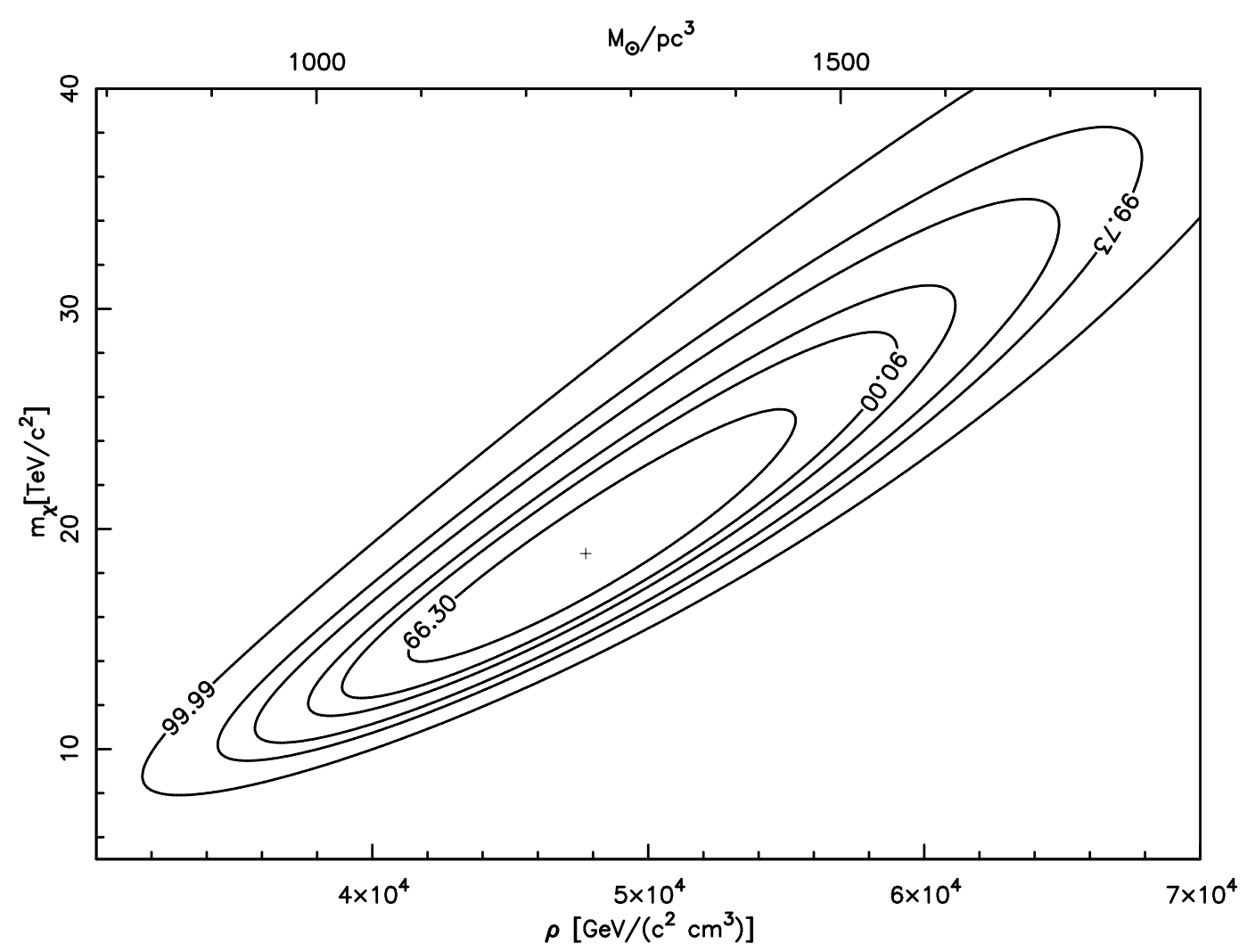

Fig. 3. For the $\chi^{2}$ fit of the model energy spectrum to the data confidence regions are indicated for different confidence values. The best-fit model is indicated with a small cross.

\section{Discussion}

TeV gamma-ray emission from the Galactic center has been observed by different different ground-based instruments. The CANGAROO group claims a signal at a significance close to $10 \sigma$ and the VERITAS group sees a indications for a signal observed in archival data. Finally, the H.E.S.S. system reported an overall excess of $11.8 \sigma$ from the Galactic center region from observations during the build-up phase.

However, taking the H.E.S.S. data alone, no indication for variability is seen and moreover, the source location is consistent with the position of $\operatorname{Sgr~A}^{*}(\Delta \theta=$ $(18 \pm 42)$ " with a systematic error of $\approx 20 ")$ and an upper limit for a Gaussian surface density source of 3 '.

Taking these results, a hypothetical Dark Matter annihilation scenario is discussed. The observed energy spectrum is reasonably well described by the continuum emission from SuSy WIMPS (neutralinos) with a mass of $18 \mathrm{TeV}$ (lower limit of $12 \mathrm{TeV}$ ) putting it far out of reach for future accelerator experiments. 


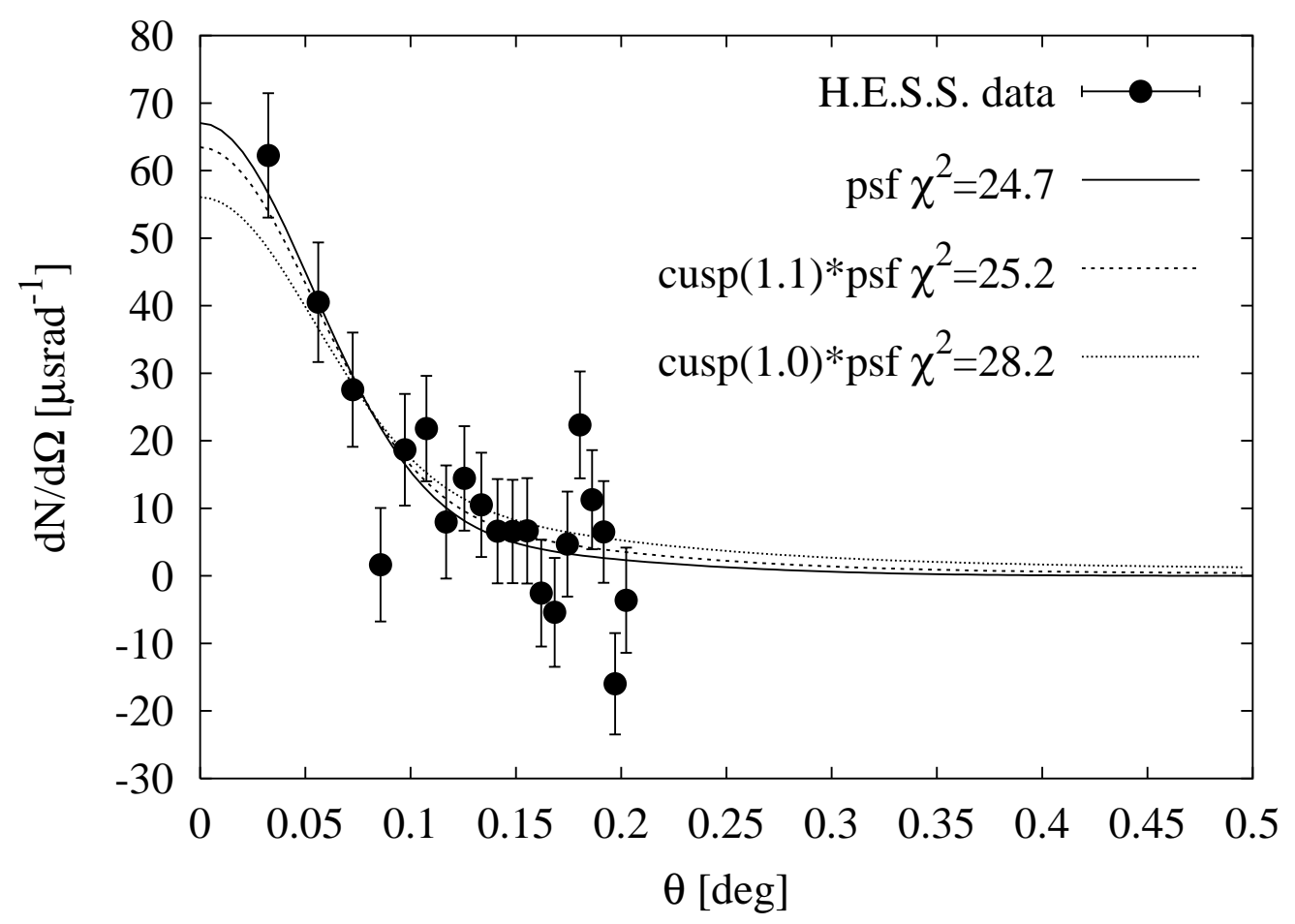

Fig. 4. For different values of the slope of the density profile, the expected observed angular distribution (after folding with the point spread function) allows to separate the case of $\alpha<1.0$ and $\alpha>1.0$. From the good agreement of data with a point source, a steep (cuspy) halo is required.

The required average Dark Matter density is $(1092 \pm 107) \cdot M_{\odot} \mathrm{pc}^{-3}$ (for $\langle\sigma v\rangle=$ $3 \cdot 10^{-26} \mathrm{~cm}^{3} \mathrm{~s}^{-1}$ and $\alpha=1$ ) for the inner $10 \mathrm{pc}$ of the Galaxy with a cuspy profile $\rho \propto r^{-\alpha}$ with $\alpha>1$ in order to fit the observed angular distribution. The upper limit on nonbaryonic Dark Matter density is close to the stellar (baryonic) mass density which is $\rho_{*}(r) \approx(1900 \pm 700) \times(r / 10 \mathrm{pc})^{-1.4 \pm 0.1} M_{\odot} \mathrm{pc}^{-3}[33]$. The total inferred mass $M(r<10 \mathrm{pc})=(3.1 \pm 0.3) \times 10^{6} M_{\odot}$ is well below the stellar mass of $(1.5 \pm 0.6) \times 10^{7} M_{\odot}$ in the inner $10 \mathrm{pc}$. The rather high central values of the density is consistent with the formation of a mini-cusp as a consequence of interaction with the central dense stellar environment $[34,35]$ and baryonic compression [36].

The angular resolution in gamma-rays achieved with H.E.S.S. allows to probe the actual shape of the Dark Matter profile for the Galactic center. With more data taken with the full telescope array operational, a further improvement on the constraint on the cuspiness is expected and test the different possibilities for the origin of the signal.

At this point, the considered annihilation scenario of WIMPS as the origin of the observed flux of gamma-rays is not ruled out. The other detectable channels of annihilation i.e. excess of positrons and anti-protons in Cosmicrays are not affected by a mini-cusp scenario as outlined above. The large 
mass of the WIMP may also produce a signal for neutrino-telescopes but the sensitivity of the current instruments is not sufficient to detect neutrinos from the Galactic center [40]. In principle, bounds from big-bang nucleosynthesis could be of importance but as outlined in [41], a very massive WIMP would not have a strong impact on abundances of light elements. Future observations of variability in the gamma-ray flux or a possible extension would be of great importance for the interpretation of the signal.

The author is grateful for discussions with F. Aharonian, J. Buckley, A. Jacholkowska, J. Primack, P. Ullio, H.J. Völk, and members of the H.E.S.S. working group on Dark Matter.

\section{References}

[1] Klypin, A., Zhao, H., \& Somerville, R. S. 2002, ApJ, 573, 597

[2] Bergstroem, L. 2000, Reports of Progress in Physics, 63, 793

[3] Bertone, G, Hooper, D., \& Silk, J. , 2004, ArXiv High Energy Physics e-prints, hep-ph/0404175

[4] Ellis, J., Hagelin, J. S., Nanopoulos, D. V., Olive, K., \& Srednicki, M. 1984, Nuclear Physics B, 238, 453

[5] Blumenthal, G. R., Faber, S. M., Primack, J. R., \& Rees, M. J. 1984, Nature, 311,517

[6] Spergel, D. N., et al. 2003, ApJ, 148, 175

[7] Bergström, L., Ullio, P., \& Buckley, J. H. 1998, Astroparticle Physics, 9, 137

[8] Stoehr, F., White, S. D. M., Springel, V., Tormen, G., \& Yoshida, N. 2003, MNRAS, 345, 1313

[9] Hooper, D. \& Wang, L. 2004, Phys. Rev. D, 69, 035001

[10] Koushiappas, S. M., Zentner, A. R., \& Walker, T. P. 2004, Phys. Rev. D, 69, 043501

[11] Evans, N. W., Ferrer, F., \& Sarkar, S. 2004, Phys. Rev. D, 69, 123501

[12] Tsuchiya, K., et al. 2004, ApJ, 606, L115

[13] Kosack, K., et al. 2004, ApJ , 608, L97

[14] Hooper, D., Perez, I. d. C., Silk, J., Ferrer, F., \& Sarkar, S. 2004, ArXiv Astrophysics e-prints, astro-ph/0404205

[15] Aharonian, F.A. et al. (H.E.S.S. coll.) submitted to A\&A

[16] Yusef-Zadeh, F. 1989, IAU Symp. 136: The Center of the Galaxy, 136, 243 
[17] Mayer-Hasselwander, H. A., et al. 1998, A\&A, 335, 161

[18] Hartman, R. C., et al. 1999, ApJS, 123, 79

[19] Hooper, D. \& Dingus, B.L. 2002, ArXiv Astrophysics e-prints, astro-ph/0210617

[20] Mori, M. 2003, in Procs. of 28th ICRC, Vol. 5, ed. T. Kajita, Y. Asaka, A. Kawachi, Y. Matsubara, and M. Sasaki (Tokyo: Universal Academy Press)

[21] Komin, N. et al. 2004, in F.A. Aharonian \& H.J. Völk (eds.) AIP Conference Series in press

[22] Berge, D. et al. 2004, in F.A. Aharonian \& H.J. Völk (eds.) AIP Conference Series in press

[23] Witten, E. 1981, Nuclear Physics B, 188, 513

[24] Feng, J. L., Matchev, K. T., \& Moroi, T. 2000, Physical Review Letters, 84, 2322

[25] Griest, K. \& Kamionkowski, M. 1990, Physical Review Letters, 64, 615

[26] Ellis, J., Olive, K. A., Santoso, Y., \& Spanos, V. C. 2003, Physics Letters B, 565,176

[27] Coleman, S. 1985, Nuclear Physics B, 262, 263

[28] Enqvist, K. \& McDonald, J. 1999, Nuclear Physics B, 538, 321

[29] Fujii, M. \& Hamaguchi, K. 2002, Phys. Rev. D, 66, 083501

[30] Navarro, J. F., Frenk, C. S., \& White, S. D. M. 1997, ApJ, 490, 493

[31] Moore, B., Ghigna, S., Governato, F., Lake, G., Quinn, T., Stadel, J., \& Tozzi, P. 1999, ApJ, 524, L19

[32] Gondolo, P., Edsjo, J., Ullio, P., Bergstrom, L., Schelke, M., \& Baltz, E. A. 2004, ArXiv Astrophysics e-prints, astro-ph/0406204

[33] Genzel, R. et al. 2003, ApJ, 595, 812

[34] Gnedin, O.Y. \& Primack, J.R. 2003, ArXiv Astrophysics e-prints, astro-ph/0308385

[35] Merritt, D. 2004, Physical Review Letters, 92, 201304

[36] Prada, F. et al. 2004, ArXiv Astrophysics e-prints, astro-ph/0401512

[37] P. Gondolo, Phys. Lett. B 494 (2000) 181

[38] R. Aloisio, P. Blasi, \& A.V. Olinto, JCAP 05 (2004) 007

[39] P. Gondolo, J. Silk, Phys. Rev. Lett. 83 (1999) 1719

[40] Bertone, G., Nezri, E., Orloff, J., \& Silk, J. 2004, ArXiv Astrophysics e-prints, astro-ph/0403322

[41] Jedamzik, K. 2004, ArXiv Astrophysics e-prints, astro-ph/0405583 